\title{
The Quantum Completeness Problem
}

\author{
Carsten Held \\ Philosophisches Seminar, Universität Erfurt, Erfurt \\ Germany
}

\section{Introduction}

Quantum mechanics (QM) is complete in a precise sense. It cannot be supplemented by more informative descriptions of physical systems given certain reasonable assumptions; this is what the no-hidden-variables proofs show. Theorems of Kochen-Specker-type (KS theorems) crucially employ an assumption of context-independence of the observables considered while those of Bell-type theorems use an assumption of locality. Since locality amounts to context-independence of local observables the former theorems can be considered as more general than the latter. What exactly do these theorems show in terms of physics? In which sense do they prove QM to be complete?

The standard answer to these questions is indeed older than the theorems themselves. It was given by the inventors of QM, notably von Neumann (who himself devised a no-hiddenvariables argument) and Dirac. It says that the mathematical entity representing the maximal QM information we can have about a system $S$ represents all the physical properties that $S$ has. This is an informative physical interpretation of the theorems. Since it embodies the idea that the QM information about $S$ is the complete representation of its physical state it may be called the completeness assumption.

In this paper, I will try to show that the completeness assumption is not the correct interpretation of the KS theorems for an ultimately simple reason: it cannot be squared with QM itself. The argument proceeds as follows. Initially, I explicate four properties of QM properties not mentioned but represented in standard axiomatisations - that will drive the argument, and moreover cast the completeness assumption into a precise form: COMP (basically a weakened version of the well-known eigenstate-eigenvalue link) (sec.2). Then I consider the central equation of the QM statistical algorithm: the trace formula. From the QM properties I conclude that the formula must be explicated in one of two ways. Neither option, however, can be harmonised with both QM and a general probability principle (sec.3). I discuss whether the argument is just an involved form of the measurement problem or else unduly neglects the notion of QM measurement - both with a negative result (sec.4). I consider a fundamental objection that indeed circumvents the argument and show that it violates one of the four QM properties (sec.5). Finally, I briefly consider what the KS theorems tell us about QM given that the argument against COMP is correct (sec.6).

\section{Preliminaries}

\subsection{Properties of quantum mechanics}

Initially, we must identify the features of QM needed for the argument. The theory can be introduced in various ways, hence comes in different versions but the four properties we 
need are shared by all of them. I choose a standard formulation of QM, using the Schrödinger picture and density operators, and illustrate everything from there but the argument in no way hinges on this choice. Suppose that the standard formulation comes as a set of axioms. The number of the axioms and their individual details will differ but the axioms will rule three important things. First, there will be an axiom fixing the mathematical entity representing the state of a QM system $S$ for a fixed time $t_{0}$ : a density operator $W\left(t_{0}\right)$ on an appropriate Hilbert space. Second, there will be an axiom ruling the time evolution of state $W\left(t_{0}\right)$ : an equation $W(t)=U(t) W\left(t_{0}\right) U(t)^{-1}$ where $U(t)=\exp [-i H t]$ is a function of time, and $\boldsymbol{H}$, possibly time-dependent $(\boldsymbol{H}=\boldsymbol{H}(\mathrm{t}))$, is a unitary operator representing $\mathrm{S}^{\prime}$ s total energy. Finally, there will be an axiom decreeing how QM generates predictions. It will either be or directly imply a statistical algorithm. Assuming a nondegenerate and discrete observable $A$ on $S$ with eigenvalues $\boldsymbol{a}_{i}$ (with i $=1,2, \ldots$ ) and $\boldsymbol{a}_{k}$ an arbitrary one of the $\boldsymbol{a}_{i}$, one form of the algorithm will include an equivalent of the following equation: $\left.\mathrm{p}\left(\boldsymbol{a}_{k}\right)=\operatorname{Tr}\left(\mathrm{W} \mathbf{P}_{a k}\right)\right)$, called the trace formula. Note that $\mathrm{p}\left(\boldsymbol{a}_{k}\right)$, on the left abbreviates $\mathrm{p}\left([A]=\boldsymbol{a}_{k}\right)$ where ${ }^{\prime}[A]=\boldsymbol{a}_{k}{ }^{\prime}$ means 'the value of $A$ on $S$ equals $\boldsymbol{a}_{k}$ ' or equivalently 'S has value $\boldsymbol{a}_{\boldsymbol{k}}$ of $\boldsymbol{A}^{\prime}$.

This little sketch of one form of QM illustrates four crucial properties that it shares with all other forms. These properties are independent of the axiomatisation details. In particular, they are independent of: a) which specific form the statistical algorithm (often called the Born Rule) takes, wherein the trace formula ultimately resides; b) whether or not we have projection in QM. The properties are dependent on the Schrödinger picture but have counterparts in the Heisenberg picture such that an argument parallel to the one given here can be constructed. (I will, however, not try to show this and leave the Heisenberg picture mostly out of discussion.) The first property is this:

Time Parameter Uniqueness: QM contains a unique time-parameter $t$.

The time-parameter in QM has a clear role to play. Given an initial value $t_{0}$ of $t$, variable values of $t$ relate states to $W\left(t_{0}\right)$ by $W(t)=U(t) W\left(t_{0}\right) U(t)^{-1}$. The variable $t$ can be assumed to take values ad libitum, but there is no second time parameter in QM. In particular, QM leaves no conceptual room for re-interpreting two occurrences of $t$ in any QM equation as two independent parameters ( $t$ and $t^{\prime}$, say) capable of taking different values. (The evolution equation $W(t)=U(t) W\left(t_{0}\right) U(t)^{-1}$ where $t$, if it takes a value, must take the same in all three places is one trivial example of this property.)

The second property is a trivial consequence of the Schrödinger picture:

Time Reference: If $Q M$ ascribes to $S$ a state $W$, then $W=W(t)$, i.e. $W$ is time-dependent throughout.

Given $\mathrm{W}=\mathrm{W}\left(\mathrm{t}_{0}\right)$, Time Reference is satisfied. Up to a measurement, all other states are produced from $W\left(t_{0}\right)$ via time evolution, so are time-dependent by construction. The situation will be different upon measurement - but only if we have a form of QM where unitary evolution is interrupted: projection. As I said, the argument below will be independent of whether or not QM contains a projection postulate because it employs just time-evolution up to a "first" measurement. But note that Time Reference is also respected, given projection, in the situation during or after a measurement. If we accept projection we assume a "collapse" of S's state upon measurement of $W\left(t_{1}\right)$. There will be an immediate problem about specifying the time-reference for collapsed state $W^{\prime}$. But collapse interpretations (like the GRW theory) usually assume that a collapsed state $\mathrm{W}^{\prime}$ can be an input for a new unitary evolution. For this to be possible, the collapsed state must also be time-dependent: $W^{\prime}=W^{\prime}\left(t_{2}\right)$ (where $t_{1}, t_{2}$ are two different values of the same parameter $\mathrm{t}$ ). 
State Uniqueness: If $\mathrm{QM}$ attributes a state $\mathrm{W}(\mathrm{t})$ to $\mathrm{S}$, then for all $\mathrm{W}^{\prime}(\mathrm{t})$ attributed to $S$, $W(t)=W^{\prime}(t)$.

This property again is a trivial consequence of our using the Schrödinger picture. QM would break down if the state attributed to $S$ per time were not unique. This would simply result in conflicting inputs for the trace formula, hence lead to conflicting predictions.

The last property relates to the trace formula $\left.\mathrm{p}\left(\boldsymbol{a}_{k}\right)=\operatorname{Tr}\left(\mathrm{W} \mathbf{P}_{a k}\right)\right)$ and may be called Generality.

Generality: Given an arbitrary state $\mathrm{W}$ of $\mathrm{S}$ and an arbitrary observable $\boldsymbol{A}$, it is the case that $\mathrm{p}\left(\boldsymbol{a}_{k}\right)=\operatorname{Tr}\left(\mathrm{W} \mathbf{P}_{a k}\right)$.

The emphasis here is on arbitrary $\mathrm{W}$ and $A$. When representing a physical system in QM, we pick a Hilbert space whose dimension depends on the observables we are interested in. Given that such a choice has been made, Generality simply says that the trace formula holds good for all states and observables on that space.

These four properties suffice to create a conflict with the completeness assumption that I call the completeness problem. Plainly, the properties are shared by all versions of QM using the Schrödinger picture and density operators. Moreover, they have counterparts in versions making other choices. I thus assume that QM as such leads to the completeness problem.

\subsection{Completeness}

KS theorems show the impossibility of assigning values to QM observables given essentially two constraints. These theorems translate the idea of assigning properties (values of observables) to $S$ into the task of assigning values to operators forming a certain algebra. Because the algebra is non-Boolean it is possible to find finite sets of operators such that not all of them can consistently be assigned the real numbers that are the allowed values of the associated observables. One condition of the arguments is that the algebra is based on a Hilbert space $\mathcal{H}$ with $\operatorname{dim} \mathcal{H}>2$. This condition is unquestionable because most QM systems require representation on a Hilbert space $\mathcal{H}$ with at least $\operatorname{dim} \mathcal{H}=3$. But there are two substantial assumptions going into the theorems that can be questioned: (1) Algebraic relations among operators representing QM observables are mirrored exactly in the associated values; this is a condition known in the literature as Functional Composition. (2) There is a one-one correspondence between observables and the operators representing them; since this can be interpreted as an assumption of independence of any single observable from the observables considered in conjunction with it this condition is generally called Noncontextuality. ${ }^{1}$ Given these two assumptions, relations among operators translate straightforwardly into relations among values of observables that cannot simultaneously be satisfied. ${ }^{2}$ Trivially, all the sets of operators are such that they cannot all have one eigenstate in common.

Generally, the completeness assumption is seen as being embodied in the so-called eigenstate-eigenvalue link $(\mathrm{EE})^{3}$ stating that $S$ has $\boldsymbol{a}_{\boldsymbol{k}}$ if and only if it is the pertaining eigenstate $\mathbf{P}_{a k}$. Following this policy we can, as a first stab, rule this equivalence:

\footnotetext{
1 See Redhead (1987, ch. 5) for a (by now classic) discussion.

2 See Kochen and Specker (1967). For a simple and unified form of the Bell and Kochen-Specker theorems for two and three particles, see Mermin (1990); see also Held (2009). For the simplest KochenSpecker type arguments in three and more dimensions see the references in Bub (1999, 118-9).

${ }^{3}$ For the expression and a clear formulation see Fine (1973), 20. The link was first proposed, however, by Dirac $(1930 / 1958,46-7)$ and von Neumann $(1932 / 1955,253)$; see Bub $(1999,29)$ for a discussion.
} 


$$
[A]=a_{k} \text { if and only if } S \text { is in } \mathbf{P}_{a k} \text {. }
$$

This is insufficient, for a formal and a physical reason. In the Schrödinger picture due to Time Reference, the state is time-dependent: $\mathbf{P}_{a k}=\mathbf{P}_{a k}(\mathrm{t})$. So in $\left(^{*}\right)$ there is an implicit time reference on the right. Now, while it is logically possible to have no matching time reference on the left this would clearly leave $\left(^{*}\right)$ incomplete. Moreover, the statement that $[A]=a_{k}$ is physically void if not referring to time concretely. In particular, we will want to tell stories such that at certain times it is true of $S$ that $[A]=a_{k}$, but not at certain others. The only natural way to make $\left.{ }^{*}\right)$ meaningful formally and physically is explication on both sides:

$$
[A]=a_{k} \text { at } \mathrm{t} \text { if and only if } S \text { is in } \mathbf{P}_{a k}(\mathrm{t}) .
$$

This is the full-fledged EE. 4 One of its directions will suffice to make sense of the KS impossibility results. The 'only if'-direction is:

$$
\text { If } S \text { is not in } \mathbf{P}_{a k}(\mathrm{t}) \text {, then } \neg[A]=a_{k} \text { at } \mathrm{t} .
$$

Now, we know (by State Uniqueness) that the state $W(t)$ of $S$ is unique, i.e. that if $S$ is in a state $\mathrm{W}(\mathrm{t}) \neq \mathbf{P}_{a k}$, then $\mathrm{S}$ is not in $\mathbf{P}_{a k}(\mathrm{t})$. Hence, from the 'only if' direction of EE:

$$
\text { If } S \text { is in a state } \mathrm{W}(\mathrm{t}) \neq \mathbf{P}_{a k} \text {, then } \neg[A]=a_{k} \text { at } \mathrm{t} \text {. }
$$

Let's weaken this one step further - to see what is minimally needed to make sense of the results. ${ }^{5}$ Assume only that when $S$ is in a pure state other than $\mathbf{P}_{a k}(\mathrm{t})$, then it does not have $\boldsymbol{a}_{\boldsymbol{k}}$ at $t$. Identify this as our completeness assumption (COMP):

$$
\text { If } S \text { is in a pure state } \mathrm{W}(\mathrm{t}) \neq \mathbf{P}_{a k} \text {, then } \neg[A]=\boldsymbol{a}_{k} \text { at } \mathrm{t} \text {. }
$$

COMP makes eminent physical sense of the KS impossibility results. Every KS set includes observables that do not all have one eigenstate in common. Whatever $S^{\prime}$ s state - if it's a pure state then the set contains observables for which it is not an eigenstate. These observables do not have values while the others may have. The contradiction that would arise from attributing values to all observables is avoided.

The crucial role of time in this reasoning should be made as explicit as possible. The starting point is that certain relations among values $[A],[B],[C] \ldots$ of certain observables $A, B, C \ldots$ (that do not all have one eigenstate in common) cannot jointly be satisfied. We want to concretize this in terms of physics in the sense that $A, B, C \ldots$ cannot all simultaneously have values. Suppose that we want to express that $A$ has a value while $B$ and $C$ have none, at the same time $t:{ }^{\prime}[A]=\boldsymbol{a}_{k}$ at $\mathrm{t}^{\prime},{ }^{\prime} \neg[A]=\boldsymbol{a}_{i}$ at $\mathrm{t}^{\prime}($ for all $i \neq k),{ }^{\prime} \neg[B]=\boldsymbol{b}_{i}$ at $\mathrm{t}^{\prime}($ for all $i),{ }^{\prime} \neg[C]=\boldsymbol{c}_{i}$ at $\mathrm{t}^{\prime}$ (for all $i$ ). An interpretive principle can imply this set of propositions only if it provides time references for the value ascriptions. COMP does just this in the only conceivable way. It uses the time reference attached to state $\mathrm{W}(\mathrm{t})$ and transfers it to negations of value ascriptions. It is hence crucial to relate these negations to time $t$ in order for COMP to explicate the interpretation of the KS results we intend.

\footnotetext{
${ }^{4}$ While I say that $\left(^{*}\right)$ must be explicated as EE to make formal and physical sense, a competing intuition has it that $(*)$ is perfectly o.k. as an expression of the eigenstate-eigenvalue link and simply implies EE. The net result is the same, of course. We are entitled to proceed with EE as the legitimate explication of the link.

${ }^{5}$ This intention can also be put another way. The full EE is not needed to make sense of the KS results. Modal interpretations reject EE (assume that if $S^{\prime}$ s state is a reduction mixture, then it is possible that $\left.[A]=a_{k}\right)$, but accept COMP. The ensuing argument creates a problem for these interpretations, too.
} 


\section{Main argument}

\subsection{Explicating the trace formula}

From Time Reference, we know that the trace formula inevitably carries a time-reference on the right: $\mathrm{p}\left(\boldsymbol{a}_{k}\right)=\operatorname{Tr}\left(\mathrm{W}(\mathrm{t}) \mathbf{P}_{a k}\right)$. This creates a problem that has been little discussed in the foundational literature. The equation has a time-reference on the right, but it is unclear whether and eventually where there is a matching one on the left. Does $\mathrm{p}\left(\boldsymbol{a}_{k}\right)$ also have a time-reference? If so it deserves to be explicated. Recall that $\mathrm{p}\left(\boldsymbol{a}_{k}\right)$ is the probability for the event referred to by proposition $[A]=\boldsymbol{a}_{\boldsymbol{k}}$ '. So we interpret $\mathrm{p}\left(\boldsymbol{a}_{i}\right)$ as a probability function (for a fixed pair of operators) from a set of propositions into [0, 1]. So we can cast the problem in these questions: Is $\mathrm{p}\left(\boldsymbol{a}_{\boldsymbol{k}}\right)$ a function of time? And if so, then in which sense is it such a function?

The first question must be answered positively because of the composition of functions. The trace formula identifies a magnitude (a probability) that is a function of propositions (attributing to $S$ the different eigenvalues of $A$ ) with another magnitude (the trace) that is a function of operators (state times measured observable) one of which in turn is a function of a certain parameter (time), hence the probability function is itself a function of the parameter (time).

Now in which sense is $\mathrm{p}\left(\boldsymbol{a}_{k}\right)$ time-dependent? Since formally it is a function there are basically two possibilities. Either this function depends on $\mathrm{t}$ implicitly - ' $\mathrm{p}\left(\boldsymbol{a}_{\boldsymbol{k}}(\mathrm{t})\right)^{\prime}$ - or explicitly - ' $\mathrm{p}\left(\boldsymbol{a}_{k}, \mathrm{t}\right)$ '. In the first case, it depends on time because its argument, the proposition ' $[A]=\boldsymbol{a}_{\boldsymbol{k}}$ ', does. The argument then, in fact is the proposition ' $[A]=\boldsymbol{a}_{k}$ at $\mathrm{t}^{\prime}$. In the second case, it depends on two arguments directly, on the proposition ' $[A]=a_{k}$ ' and on time $\mathrm{t}$. For a clearer visual distinction I rewrite this second option as ' $\mathrm{p}(\mathrm{t})\left(\boldsymbol{a}_{k}\right)^{\prime}$. Schematically then the explication must be either ' $\mathrm{p}\left(\boldsymbol{a}_{k} \underline{(\mathrm{t})}\right)$ ' or ' $\mathrm{p}(\mathrm{t})\left(\boldsymbol{a}_{k}\right)$ '. Of course, the index could also be in both places at once. I ignore this third possibility here but consider it below (in sec. 3.3). We have just seen that the index must turn up somewhere and these are the two minimal possibilities. For obvious reasons the first reading can be called the internal interpretation:

$$
\mathrm{p}\left(\boldsymbol{a}_{k}(\mathrm{t})\right)=\operatorname{Tr}\left(\mathrm{W}(\mathrm{t}) \mathbf{P}_{a k}\right) .
$$

and the second the external interpretation:

$$
\mathrm{p}(\mathrm{t})\left(\boldsymbol{a}_{k}\right)=\operatorname{Tr}\left(\mathrm{W}(\mathrm{t}) \mathbf{P}_{a k}\right) .
$$

These two are the only apparent choices. Without arguing for it further, I assume that there are no others.

\subsection{The internal interpretation}

I imagine that QM can be condensed into a number of theorems and, if applied to a certain system, a number of state specifications as necessary. I then can assume QM (applied to a certain system) to be a conjunction of theorems and state specifications. I can also speak of $\mathrm{QM}$ as a conjunct in a conjunction and henceforth will speak of the conjunction of QM and COMP (applied to system $S$ with observable $A$ ) as complete QM.

I begin with a fundamental idea about probability that I hope finds universal acceptance: Probability is quantified possibility. More precisely: If a physical theory assigns an event a non-zero probability, then, given the theory's truth, this event is possible. The weakest form 
of possibility is logical possibility. Using it we can implement the idea in the following probability principle: If a theory $\mathrm{T}$ for a proposition $\mathrm{F}$ (describing an event) yields another proposition $p(F)>0$ then it must be compatible with $F$, i.e. it is not the case that the set $\{T, F\}$ makes derivable a contradiction (in first-order logic, I shall assume).

Now suppose that $S$ is in a pure state $W\left(t_{1}\right) \neq \mathbf{P}_{a k}$, for some value $t_{1}$ of $t$, such that for the probability calculated from the trace formula it is the case that $1>\mathrm{p}\left(\boldsymbol{a}_{k}\right)>0$. (This is a state specification such that $S$ is in a pure non-eigenstate of $A$ at $t_{1}$.) From COMP, we know that in state $\mathrm{W}\left(\mathrm{t}_{1}\right) \neq \mathbf{P}_{a k} \mathrm{~S}$ does not have value $\boldsymbol{a}_{k}$ at $\mathrm{t}_{1}$, i.e. $\neg[\mathrm{A}]=\boldsymbol{a}_{k}$ at $\mathrm{t}_{1}$. But from the internal interpretation we know that $\mathrm{p}\left(\boldsymbol{a}_{k}\right)=\mathrm{p}\left(\boldsymbol{a}_{\boldsymbol{k}}\left(\mathrm{t}_{1}\right)\right)$ which is the probability that $[\mathrm{A}]=\boldsymbol{a}_{\boldsymbol{k}}$ at $\mathrm{t}_{1}$. This probability is strictly positive, hence the theory producing it should be compatible with $[\mathrm{A}]=a_{k}$ at $\mathrm{t}_{1}-$ which complete QM evidently is not (given the state specification in question). Contradiction. It would not be correct to say that the internal interpretation contradicts COMP. In fact, the correct thing to say is that complete QM (including a trivially admissible state specification) plus the internal interpretation contradicts the probability principle. I trust that the reader finds this principle as fundamental as I do. So, the internal interpretation is untenable in complete QM. ${ }^{6}$

\subsection{The external interpretation}

There is only one tangible alternative and hence only one conclusion to be drawn. Since the internal interpretation is in conflict with complete QM we must employ the external interpretation to make sense of it.

We must consider (briefly) the following idea. Assuming that the time-parameter, in the external interpretation, refers to the probability for an event (not the event itself) we might still be free to assume that the parameter also refers to the event. Explicitly, the probability calculated from the trace formula, given a state $\mathrm{W}\left(\mathrm{t}_{1}\right)$, might be $\mathrm{p}\left(\mathrm{t}_{1}\right)\left(\boldsymbol{a}_{k}\left(\mathrm{t}_{1}\right)\right)$. Obviously, this is not a helpful idea because we now have a strictly positive probability at $t_{1}$ for S having $\boldsymbol{a}_{k}$ at $\mathrm{t}_{1}$, i.e. for $[\mathrm{A}]=\boldsymbol{a}_{k}$ at $\mathrm{t}_{1}$. But we also still have, from $\mathrm{W}\left(\mathrm{t}_{1}\right) \neq \mathbf{P}_{a k}\left(\mathrm{t}_{1}\right)$ and COMP, that $S$ does not have value $a_{k}$ at $t_{1}$, i.e. $\neg[\mathrm{A}]=a_{k}$ at $\mathrm{t}_{1}$. So, there still is a contradiction with our probability principle. The only way out is to assume that the time-parameter (here its value $t_{1}$ ), which, given the external interpretation, the probability inherits from the state $\mathrm{W}\left(\mathrm{t}_{1}\right)$, does not also refer to the event for whose realization the probability is given. What it does refer to is only the probability itself.

However, we know from Time Parameter Uniqueness that QM contains just one parameter $t$. If it is not this parameter whose values are time-references for the predicted events in the trace formula, then these events can carry no time-references, at all. Hence, the expression ' $\boldsymbol{a}_{\boldsymbol{k}}$ ' in the formula ' $\mathrm{p}(\mathrm{t})\left(\boldsymbol{a}_{k}\right)=\operatorname{Tr}\left(\mathrm{W}(\mathrm{t}) \mathbf{P}_{\boldsymbol{a}}\right)$ ' is necessarily timeless in complete QM. Note also the following fact. The trace formula can be suitably generalised to encompass discrete and continuous observables. The relevant counterpart of proposition ' $[\mathrm{A}]=\boldsymbol{a}_{\boldsymbol{k}}$ ' in that generalised form must of course also be timeless to match the discrete case. But then this must also hold for the continuous case, i.e. for a continuous observable $A$, a value $a$ and an arbitrary Borel subset B of the real line it must be the case that:

$$
\mathrm{p}(\mathrm{t})(a \in \mathrm{B})=\operatorname{Tr}\left(\mathrm{W}(\mathrm{t}) \mathbf{P}_{A}(\mathrm{~B})\right)
$$

where the proposition ' $a \in \mathrm{B}^{\prime}$ is necessarily timeless.

${ }^{6}$ See Held (2008, sec.s 2 and 3) for an earlier version of this argument. 
Serious problems ensue. Familiar interpretations of QM notions cannot be maintained and important applications collapse. E.g., the so-called conventional interpretation of the wavefunction turns out to be false in complete QM. However, modern approaches to QM gravity (reasonably) construct probabilities for spacetime events that have this interpretation as a limiting case, so these approaches are also endangered. Similarly, QM transition probabilities and propagators cannot be defined in their usual textbook ways. ${ }^{7}$ These problems should convince us right away that the external interpretation is not the one we use in real-life QM. Of course, the factual practice of QM does not constitute a knock-down objection in matters foundational but indeed such an objection is comparatively easy to come by. We can, with standard QM techniques, construct probabilities that carry an explicit internal time-reference - despite the fact that complete QM now forbids it.

The idea is as follows. In eq. (3.1) set $A=X$ (the observable position), $\mathrm{a}=\mathrm{x}$ and $\mathrm{B}=\Delta \mathrm{x}$ (a value and value range of $X$ ). Proposition ' $x \in \Delta x^{\prime}$ cannot carry the index $t$. And as in the discrete case this entails that the proposition cannot carry an index at all. Instead, the probability itself is time-indexed, i.e. we have another instance of the external interpretation:

$$
\mathrm{p}(\mathrm{x} \in \Delta \mathrm{x})=\mathrm{p}(\mathrm{t})(\mathrm{x} \in \Delta \mathrm{x}) .
$$

Consider now an observable that is a function of position, e.g. S's potential energy $V(x)$ and assume it to be explicitly time-dependent, i.e. $V(x, t)$. (Since the Hamiltonian $H$ is a function of $V$, it is here that we use the possibility that $\boldsymbol{H}=\boldsymbol{H}(\mathrm{t})$.) Since $\mathrm{V}$ is a function of position (we can measure it via position measurements), we can exploit the Statistical Functional Composition Principle (a direct consequence of the trace formula; see Redhead $1987,18)$ to construct probabilities for values $V$ of $V(x, t)$ and equate them with those for position:

$$
\mathrm{p}(\mathrm{V} \in \Delta \mathrm{V}(\mathrm{t}))=\mathrm{p}(\mathrm{x} \in \Delta \mathrm{x})
$$

(where $\Delta V(t)$ is time-dependent because $V(x, t)$ is). The values $V$ of $V(x, t)$ on the left are manifestly time-dependent. ${ }^{8}$ We thus have constructed a probability that explicitly has an internal time-reference and identified it with one that, given the external interpretation tailored to complete QM, cannot have such an internal time-reference. Contradiction, again.

So the external interpretation of the trace formula cannot be correct, either. We need timereferences for the events predicted by the trace formula to do sensible QM. But the internal interpretation was so easily brought into a conflict with complete QM that it seems implausible to maintain. So in the presence of COMP, neither interpretation is tenable and there is no obvious third possibility. The argument, at this point, is inescapably turning against COMP. But COMP is our best (at any rate, most generally accepted) shot at how to interpret the KS no-hidden variables results. So we should very thoroughly look at possible objections. In the next section, I will consider whether the argument is a variant of the infamous measurement problem or otherwise trades on a neglect of the notion of QM measurement. Neither will turn out to be the case. I will then look at the only clear objection to the argument.

\footnotetext{
${ }^{7}$ Both these claims are substantiated in a companion paper (Held, in preparation).

${ }^{8}$ Details of the construction are again given in Held (in preparation).
} 


\section{Measurement}

\subsection{The measurement problem}

An obvious intuition about the previous argument is that it constitutes just a peculiar form of the measurement problem and it is old wisdom that any interpretation of QM endorsing COMP must try to deal with that difficulty. But only the second half of this intuition is correct, not the first. Indeed it is true that a formulation of the measurement problem, in general, presupposes COMP. Let me quickly recall this.

For simplicity, assume that observable $A$ on $S$ has just two distinct eigenvalues $\boldsymbol{a}_{1}$ and $\boldsymbol{a}_{2}$. Let $S$ be measured by an $A$-measurement apparatus $M$ and let $\left[\mathrm{t}_{1}, \mathrm{t}_{3}\right]$ be an interval such that $t_{1}$ is the time of measurement onset and $t_{3}$ some later time. Assume that $S$ is in a superposition of $A$-eigenstates, e.g. in $\mathbf{P}_{\boldsymbol{b}}\left(\mathrm{t}_{1}\right)=|\boldsymbol{b}\rangle\langle\boldsymbol{b}|$ where $|\boldsymbol{b}\rangle=1 / \sqrt{2}\left(\left|\boldsymbol{a}_{1}\right\rangle+\left|\boldsymbol{a}_{2}\right\rangle\right)$. First, assume that from our observation of a suitable pointer observable $\boldsymbol{M}$ on $\mathrm{M}$ we learn that $[M]=m_{1}$ at $t_{3}$. From this we want to conclude something about $S^{\prime}$ s value of $A$, e.g. $[A]=a_{1}$ at $t_{2}-$ where $t_{2}$ is an arbitrary time from $\left[t_{1}, t_{3}\right]$, including the boundaries. All choices of $t_{2}$ are perfectly possible. In particular, it is consistent to assume jointly that $S$ is in $\mathbf{P}_{\boldsymbol{b}}\left(\mathrm{t}_{1}\right)$ (a projector onto a non-eigenstate of $A$ ) and $[A]=\boldsymbol{a}_{1}$ at $\mathrm{t}_{1}$. Similarly, if $S$ is in $\mathbf{P}_{\boldsymbol{b}}\left(\mathrm{t}_{2}\right)$, where $t_{2}$ is an arbitrary time with $\left.t_{2} \in\right] t_{1}, t_{3}$, , ( $S$ stays in the superposition due to unitary time evolution) we can consistently assume $[A]=\boldsymbol{a}_{1}$ at $\mathrm{t}_{2}$. Now, assume also EE plus State Uniqueness (from sec.2.1) or alternatively COMP. Then on pain of contradiction, we can assume jointly neither $\mathbf{P}_{\boldsymbol{b}}\left(\mathrm{t}_{1}\right)$ and $[A]=\boldsymbol{a}_{1}$ at $\mathrm{t}_{1}$ nor $\mathbf{P}_{\boldsymbol{b}}\left(\mathrm{t}_{2}\right)$ and $[A]=\boldsymbol{a}_{1}$ at $\mathrm{t}_{2}$. We can no longer draw any conclusions from our assumed observation $[M]=m_{1}$ at $t_{3}$ to $S$ - which is what generally a measurement of $S$ by means of $M$ is intended to deliver.

In its more familiar form, the measurement problem refers not to conclusions from observed pointer values to $S$ values but instead to the $Q M$ state of the apparatus $M$. Let an $A$-measurement on $S$ by $\mathrm{M}$ be modelled in the combined system $\mathrm{S}+\mathrm{M}$ by initial states $\mathbf{P}_{R i}\left(\mathrm{t}_{1}\right)=\left|\mathrm{R}_{\mathrm{i}}><\mathrm{R}_{\mathrm{i}}\right|$, where $\left|\mathrm{R}_{\mathrm{i}}\right\rangle=\left|\boldsymbol{a}_{i}>\right| \boldsymbol{m}_{\text {ready }}>$ and final states $\mathbf{P}_{A i}\left(\mathrm{t}_{2}\right)=\left|\mathrm{A}_{\mathrm{i}}><\mathrm{A}_{\mathrm{i}}\right|$, where $\left|A_{i}>=\right| a_{i}>\mid m_{i}>$, for $\mathrm{i}=1,2$, and where $\left.\left.t_{2} \in\right] \mathrm{t}_{1}, \mathrm{t}_{3}\right]$. Then, if $\mathrm{S}+\mathrm{M}$ is in state $\mathbf{P}_{R}\left(\mathrm{t}_{1}\right)=|\mathrm{R}\rangle\langle\mathrm{R}|$, where $|\mathrm{R}\rangle=|\boldsymbol{b}\rangle \mid \boldsymbol{m}_{\text {ready }}>$ and $|\boldsymbol{b}\rangle$ as before, it follows that $\mathrm{S}+\mathrm{M}$ is in state $\mathbf{P}_{B}\left(\mathrm{t}_{2}\right)=|\mathrm{B}><\mathrm{B}|$ with $\left|\mathrm{B}>=1 / \sqrt{2}\left(\left|\boldsymbol{a}_{1}\right\rangle\left|\boldsymbol{m}_{1}\right\rangle+\left|\boldsymbol{a}_{2}\right\rangle\left|\boldsymbol{m}_{2}\right\rangle\right)\right.$. Again, it is perfectly consistent to assume that $S+M$ is in $\mathbf{P}_{B}\left(t_{2}\right)$ and $[M]=m_{1}$ at $t_{2}$ (or alternatively $[M]=m_{1}$ at $\left.t_{2}\right)$. Now, assume EE in the form: $[M]=m_{i}$ at $t$ if and only if $S$ is in $\mathbf{P}_{m i}(t)($ for $\mathrm{i}=1,2)$ plus again State Uniqueness. Then, again on pain of contradiction, we can assume jointly neither $\mathbf{P}_{B}\left(t_{2}\right)$ and $[M]=m_{1}$ at $t_{2}$ nor $\mathbf{P}_{B}\left(t_{2}\right)$ and $[M]=m_{2}$ at $t_{2}$ for any $\left.\left.t_{2} \in\right] t_{1}, t_{3}\right]$. The apparatus has been pushed into a QM state such that, given EE, it is impossible to assume it as showing one of the pointer values - in contradiction with what we really observe. (As is well-known, COMP here is too weak to generate the contradiction because $\mathrm{M}^{\prime}$ s state at $t_{2}$ is a reduction mixture. As is also well-known, moving from EE to COMP alone does not suffice to avoid the measurement problem since the $M$ reduction mixture cannot be given an ignorance interpretation.)

So, the measurement problem confronts QM states and hypothetical observations by means of EE plus State Uniqueness or COMP - which means that it presupposes EE or COMP. The argument of sec. 3 is not a form of the measurement problem simply because it does not mention any observations at all that could be confronted with QM states. On the other hand, the measurement problem requires COMP or the stronger EE for its formulation. And the argument of sec.3 shows that COMP is incompatible with QM, even without that confrontation. If sound the argument provides grounds to reject the measurement problem 
because it rests on a combination of COMP (or EE) and QM, which is in a deeper sense incoherent, i.e. leads to a consistency problem without invoking observations (though using other assumptions).

\subsection{The value assumption}

Now, while the previous argument cannot be said to be a version of the measurement problem one may still think that it unduly neglects the crucial role of measurement in QM. I will try to dispel this impression. Evidently, the argument has not mentioned measurement. This would be inevitable if the two readings of the trace formula were integrated into a full axiom system for QM. Such a system would contain a statistical algorithm and that algorithm would be (or directly entail) one for generating probabilities via the trace formula. In formulating the latter algorithm - the Born Rule - we would have to take a stand on the notion of measurement. Generally, measurements and outcomes are mentioned explicitly and many think that this is an unavoidable feature of the Born Rule. ${ }^{9}$ But considering the appropriate wording of the Born Rule here would just needlessly complicate the argument - which requires nothing but the trace formula itself. ${ }^{10}$

Let me however explicate an assumption that has tacitly been made in the argument (and will be tentatively questioned below, in sec.5). Recall that in the trace formula I took $\mathrm{p}\left(\boldsymbol{a}_{\boldsymbol{k}}\right)$ to be the probability for $[A]=a_{k}$, i.e. explicitly for 'S has value $a_{k}$ of $A^{\prime}$ '. The argument in sec.3.2 would not get off the ground if $\mathrm{p}\left(\boldsymbol{a}_{k}\right)$ were not this probability for $[A]=a_{k}$, because the conflict arises between $\mathrm{p}\left(\boldsymbol{a}_{\boldsymbol{k}}\right)>0$ (from the trace formula and an admissible state specification) and $\neg[A]=\boldsymbol{a}_{k}$ (from COMP). The conflict becomes sharp, if both statements are nailed to the same time and we assume that $\mathrm{p}(\mathrm{F})>0$ entails that $\mathrm{F}$ can consistently be added as an assumption (our probability principle). But even then a contradiction will only arise if in 'p $\left(\boldsymbol{a}_{k}\right)$ ' the "inner" expression ' $\boldsymbol{a}_{\boldsymbol{k}}$ ' really means 'S has value $\boldsymbol{a}_{k}$ of $\boldsymbol{A}^{\prime}$. If that is not the case then even fixing the same time for both propositions will not generate any problem. So the assumption at work in the previous argument is that in the trace formula:

$$
\mathrm{p}\left(a_{k}\right)=\mathrm{p}\left([A]=a_{k}\right),
$$

i.e. $\mathrm{p}\left(\boldsymbol{a}_{k}\right)$ is the probability that $S$ has value $\boldsymbol{a}_{k}$ of $A$.

This very elementary assumption may be called the value assumption because it amounts to the claim that the events concerning S, for which QM encodes probabilities, are simply identical to $S$ having a value of an observable. Note carefully that this assumption does not involve a claim about when $S$ eventually has value $\boldsymbol{a}_{k}$ of $\boldsymbol{A}$ and hence is not per se in a conflict with certain widespread intuitions about QM probabilities. Instead it helps to disentangle these intuitions - because we can separate the question of what the QM events ("inside" the probability of the trace formula) are from the one when they eventually occur.

I will consider three intuitions about measurement. First, there is the intuition that QM probabilities concern events that are conditional upon measurement. We can implement this in QM in different ways. First of all, we can write the following version of the Born Rule: If $S$ is in state $W(t)$ and $A$ is an observable on $S$ and if an $A$-measurement on $S$ is made then: $\mathrm{p}\left(\boldsymbol{a}_{k}\right)=\operatorname{Tr}\left(\mathrm{W}(\mathrm{t}) \mathbf{P}_{a k}\right)$. Here measurement is mentioned as one of several conditions for the

9 Among many possible references see Redhead $(1987,8)$, Hughes $(1989,70)$, van Fraassen $(1991,142-$ 143), Albert (1992, 35), Bub (1999, 31), Nielsen \& Chuang (2000, 84-85), Landsman $(2009,66)$.

${ }^{10}$ In this sense, the version of the argument of sec.3.2 given in Held (2008) is inferior to the present one. 
trace formula which itself remains formally untouched. In particular, no reason emerges for assuming that the event for which a probability (given the conditions) is encoded should not be just that $S$ has value $\boldsymbol{a}_{k}$ of $A$. Alternatively, we could insist that this special condition be directly written into the trace formula itself. Now, it is well-known that if conditional probabilities are introduced via the familiar Kolmogorov definition, then a conditional probability p $\left(\boldsymbol{a}_{\boldsymbol{k}} \mid \mathrm{M}_{\mathrm{A}}\right)$, where $\mathrm{M}_{\mathrm{A}}$ is the "event" of an $A$-measurement being initiated, is not generally well-defined. ${ }^{11}$ Suppose nevertheless, for a moment, that QM probabilities were simple conditional probabilities. Again, the value assumption could stay essentially unaffected. Probability $\mathrm{p}\left(\boldsymbol{a}_{k}\right)$ would now equal $\mathrm{p}\left(\boldsymbol{a}_{\boldsymbol{k}} \mid \mathrm{M}_{\mathrm{A}}\right)$ but in that latter expression the component ' $\boldsymbol{a}_{\boldsymbol{k}}$ ' could keep its meaning. If, on independent grounds we can manage to nail the ' $a_{k}$ ' in $\mathrm{p}\left(\boldsymbol{a}_{k} \mid \mathrm{M}_{\mathrm{A}}\right)$ to the same time $\mathrm{t}$, for which an $A$-value is prohibited via COMP, the conflict is just the same as before. Finally, consider $\mathrm{p}\left(\boldsymbol{a}_{\boldsymbol{k}}\right.$ given $\left.\mathrm{M}_{\mathrm{A}}\right)$, where 'given' is a conditional connective awaiting further analysis. ${ }^{12}$ The situation will still be the same. Appreciating that QM probabilities are conditional on measurement will not in itself contradict the assumption that the conditioned events just consist in $S$ having a value of an observable. All in all, conditioning on measurement is not in itself, in conflict with the value assumption.

Second, there is a strong intuition that, because QM probabilities are conditional on measurement, the events they concern are measurement outcomes. What is the consequence of this? Again, nothing in itself prohibits that a measurement outcome be reported in the statement ' $S$ has value $\boldsymbol{a}_{k}$ of $A^{\prime}$ '. Indeed, it is not entirely implausible to assume that a measurement outcome just is a value possessed (at some time) conditional on a measurement (at an earlier time). So, we can call the events in question by the name of outcomes and still respect the value assumption. Admittedly, the notion of an outcome is ambiguous enough to be interpreted, alternatively, as not meaning a possessed value but something else. This ambiguity, however, should tell against a use of "outcome" in the foundations of QM without clarifying whether or not an outcome is a (perhaps conditionally) possessed value of $S$ or not.

Finally, it is sometimes said that QM systems upon measurement display or show values of observables $^{13}$ and hence QM probabilities should be interpreted as concerning such displaying or showing events. Since displaying or showing something is representing something, this is just a terribly misplaced metaphor. But even if we take QM systems as representing something upon measurement, there still are only two options. Does $S$ have or possess what it represents (or displays or shows) upon measurement? If so the value assumption is met, if no it isn't. Again, the idea in itself is ambiguous enough to be interpreted in a sense that is not in conflict with that assumption.

Before moving on I describe a simple non-QM example where all three intuitions plus the value assumption are respected. Our present picture of QM measurement may be described by using the following analogy. Such measurement is not like opening a box and determining the colour of the ball inside. Instead, it is like rolling a die and recording the result. Consider this latter picture. Assume that we have a fair die and want to define probabilities for the experiment of rolling it. We understand that the type of event, for which we define probabilities, is that the die shows a certain one of its six faces at the end of the

11 See van Fraassen and Hooker (1976), 229.

12 See Halpin (1991), Butterfield (1993), Held (2008, sec.5) for more on the appropriate analysis.

13 See Earman's portrayal of a 'non-realist' interpretation of QM measurement in Earman (1986), 219. 
experiment. The probability $\mathrm{p}(6)=1 / 6$ is directly understood as being the probability at the start of the experiment and as being conditional upon actually rolling the die. If measuring what the die shows is indeed doing the experiment of rolling it then, if the die in the end shows 6, that event is indeed conditional upon measurement. That event is also naturally described as the experiment's outcome and the die can meaningfully be said to display or show the number 6. Yet, there can be no doubt that the die in the end also has the value 6 (of the observable 'which face is up'). It would be confused here to assume that outcomes are not possessed values. Yes, they are but they are not values possessed initially - in contrast with what we assume about the ball in the box. The whole burden of distinguishing the die "measurement" from the "classical" colour measurement in the ball is carried by implicit time-references. In particular, in the case of the die p (6) $=1 / 6$ is the measurement outcome probability at the beginning of the experiment and the eventual outcome 6 is an event at its end. Accordingly, I now turn to a school of thought about QM measurement where probability and QM event refer to different times but the value assumption is respected. I will not try to be exhaustive but only consider two prominent authors representing that school, one a founding father of QM (von Neumann), the other a leading contemporary interpreter (van Fraassen).

\subsection{Von Neumann's account}

As has been pointed out many times before, Dirac and von Neumann can be credited with having first assumed EE - even if only implicitly. ${ }^{14}$ In von Neumann's formulation of the Born Rule the key expression is that, given a state $\mathrm{W}$ and an observable $A, \mathrm{p}\left([A]=\boldsymbol{a}_{k}\right)$ (or equivalently p $\left(\left[\mathbf{P}_{a k}\right]=1\right.$ ) is the probability that $A$ (or $\mathbf{P}_{a k}$ ) 'takes on' a value $\boldsymbol{a}_{k}$ (or 1) (see von Neumann 1932/1955, 200, 206, 211). There is only one sentence, however, where he explicitly says that the Born Rule 'does not tell us what values [the observables] have in the state W, but only with what probability they take on all possible values' (ibid., 206; my italics and notation). (In passing, I note that von Neumann does not explicitly claim this taking-on to be conditional on measurement.) Only here we explicitly learn that 'taking on' a value is opposed to 'having' it, i.e. that 'taking on' is a dynamical process. ${ }^{15}$ By a dynamical process I mean a transition in time from $S$ having no value of $A$ to having one of the $\boldsymbol{a}_{i}-$ which (since von Neumann accepts EE) simultaneously is a transition from $W \neq \mathbf{P}_{a i}$ (for any i) to $\mathbf{P}_{a i}$ (for some i): the famous "collapse of the wave-function". As that latter expression suggests, the transition is construed best as taking place instantaneously; at any rate, this is the standard understanding.

What are we to say about this instantaneous transition? Suppose, quite unreasonably, that it is instantaneous in the sense of taking up no interval of time, at all. Because of Time Reference (also explicitly endorsed by von Neumann; see 1932/1955, 198) W $=W(t)$. Now, are we to think that the transition is from $\mathrm{W}(\mathrm{t}) \neq \mathbf{P}_{a i}$ (for any i) to $\mathbf{P}_{a i}$ (for some i), where $\mathbf{P}_{a i}=\mathbf{P}_{a i}(\mathrm{t})$ ? Given State Uniqueness, this is contradictory. So this interpretation is implausible. Suppose, more reasonably, that the transition is instantaneous in the sense of

\footnotetext{
${ }^{14}$ See note 3 above.

15 The other two passages are ambiguous. Suppose that for a function $F(t)$ of parameter $t$, a real number $f$ and value $t_{1}$ of $t, F\left(t_{1}\right)=f$. One might describe this by saying that $F$ takes on value $f$ at $t_{1}$. But of course $F$ can simultaneously be said to have value $\mathrm{f}$ at $\mathrm{t}_{1}$. Accordingly, the taking-on here is not a dynamical process. This interpretation, though implausible in a physics context, is at least possible for the passages on p. 200 and p. 211.
} 
taking up an infinitesimal interval $\mathrm{dt}$ - where, as usual, "infinitesimal" means arbitrarily small but finite. Then the instantaneous transition can be viewed as being one from $W(t)$ to $\boldsymbol{P}_{a i}\left(\mathrm{t}+\mathrm{dt}\right.$ ) (for some i) and from $\mathrm{S}$ having no value of $A$ at $\mathrm{t}$ to having one of the $\boldsymbol{a}_{i}$ at $\mathrm{t}+\mathrm{dt}-$ a perfectly consistent interpretation of von Neumann's idea. But notice that $[t, t+d t]$ though arbitrarily small is finite and cannot go to zero (on pain of reproducing the previous contradiction).

Now, should von Neumann be interpreted as proposing a version of the Born Rule for transitions or for the values possessed at the end of such transitions? Naively, it seems legitimate to equate a probability for $S$ taking on a value of $A$ in the interval $[\mathrm{t}, \mathrm{t}+\mathrm{dt}]$ as the probability for $\mathrm{S}$ having that value of $A$ at $\mathrm{t}+\mathrm{dt}$ but is this interpretation admissible here? Indeed, it is not only legitimate but a necessity. Clearly, if $\mathrm{W}(\mathrm{t})=\mathbf{P}_{a k}$, then $\mathrm{p}\left(\left[\mathbf{P}_{a k}\right]=1\right.$ at $\mathrm{t}+\mathrm{dt}$ ) cannot be the probability for $\mathrm{S}$ taking on $\boldsymbol{a}_{k}$ within $[\mathrm{t}, \mathrm{t}+\mathrm{dt}]$ for that would entail that at $\mathrm{t} S$ does not have $\boldsymbol{a}_{k}$ at $\mathrm{t}-$ which contradicts EE. But Generality requires that we give the trace formula a uniform interpretation (either transitions or possessed values) for all states. Hence, it must be possessed values. One must interpret von Neumann's Born probabilities as being for the events of S having that value of $A$ at $\mathrm{t}+\mathrm{dt} .{ }^{16}$ This is to say that von Neumann must endorse the value assumption. ${ }^{17}$ Moreover, he must endorse the following version of the trace formula:

$$
\mathrm{p}\left(\left[\mathbf{P}_{a k}\right]=1 \text { at } \mathrm{t}+\mathrm{dt}\right)=\operatorname{Tr}\left(\mathrm{W}(\mathrm{t}) \mathbf{P}_{a k}\right)
$$

(where $\mathrm{p}\left(\left[\mathbf{P}_{a k}\right]=1\right.$ at $\left.\mathrm{t}+\mathrm{dt}\right)=\mathrm{p}\left([A]=\boldsymbol{a}_{k}\right.$ at $\left.\mathrm{t}+\mathrm{dt}\right)$. But why should an interpreter propose this? For an answer, I can only refer back to sec. 3.2. Von Neumann endorses COMP (because he assumes EE and certainly endorses State Uniqueness). In the presence of COMP, $\mathrm{p}\left(\left[\mathbf{P}_{a k}\right]=1\right.$ at $\left.\mathrm{t}\right)=\operatorname{Tr}\left(\mathrm{W}(\mathrm{t}) \mathbf{P}_{a k}\right)$ looks implausible (for $\mathrm{W}(\mathrm{t}) \neq \mathbf{P}_{a i}$, for any i) and indeed, given the probability principle, does yield a contradiction. It is just natural to avoid the threat of that contradiction by forcing apart time references for state and possible value.

However, against this line of thought the second part of the above argument (sec.3.3) now is seen to have bite. If (4.1) is the proposed explication of the trace formula for a state $W(t)$ and observable $\mathbf{P}_{a k}$ then this proposal is in trouble. Obviously, a value of the time-reference $t+d t$ on the left cannot come from $W(t)$ because $t$ and $t+d t$ are supposed to take different values while the size of $\mathrm{dt}$ is nowhere determined. (Recall that the infinitesimal size of $\mathrm{dt}$

\footnotetext{
${ }^{16}$ Explicitly, the argument is this. Assume that $\mathrm{p}\left(\mathrm{T}\left(\boldsymbol{a}_{i}\right)\right)=\operatorname{Tr}\left(\mathrm{W}(\underline{\mathrm{t}}) \mathbf{P}_{a i}\right)$ where $\mathrm{T}\left(\boldsymbol{a}_{\boldsymbol{i}}\right)$ is the transition from $\mathrm{S}$ not having $a_{i}$ at $\mathrm{t}$ to $\mathrm{S}$ having $\boldsymbol{a}_{i}$ at $\mathrm{t}+\mathrm{dt}$ (for all i). Presupposing Generality, assume $\mathrm{W}(\mathrm{t})=\mathbf{P}_{a k}(\mathrm{t})$. Hence, $\mathrm{S}$ is in the eigenstate pertaining to $\boldsymbol{a}_{k}$ at $\mathrm{t}$. Then by EE, S has $\boldsymbol{a}_{k}$ at $\mathrm{t}$. But the p (T $\left.\left(\boldsymbol{a}_{i}\right)\right)$ have just been defined as probabilities for transitions starting from $S$ not having $\boldsymbol{a}_{i}$ (for all i). Hence, $S$ does not have any of the values $\boldsymbol{a}_{i}$ at $\mathrm{t}$ and a fortiori not $\boldsymbol{a}_{k}$. Contradiction. In words: von Neumann's Born probabilities cannot concern transitions from no value of $A$ to value of $A$, in general, on pain of violating EE, when $\mathrm{W}$ projects onto an eigenstate of $A$.

${ }^{17}$ In fact, at a much later point von Neumann in a sense denies the value assumption when he writes: 'Indeed experience only makes statements of this type: an observer has made a certain (subjective) observation; and never any like this: a physical quantity has a certain value.' (von Neumann 1932/1955, 420). This idea is a denial of the value assumption if we assume that QM probabilities concern what we experience rather than what is the case. Note however that the present context is different because it speaks not of experience but of what is the case: von Neumann's decree that the trace formula fixes 'with what probability [observables] take on ... possible values' (206) implies that such taking-on of a value can happen. If it does then, as a result, the observable has a value - regardless of whether an observer has a mere '(subjective) observation' of that or more.
} 
does absolutely no work here. The interval cannot be set to zero without reproducing the previous contradiction, so the limit of making dt ever smaller simply does not exist. But which time value then is $t+d t$ ?) So there is nothing at all in QM to provide a value of $\mathrm{t}+\mathrm{dt}$ on the left because (due to Time Parameter Uniqueness) there is no second timeparameter in QM. Hence (4.1) cannot be a formula of QM. Instead the outcome $\left[\mathbf{P}_{a k}\right]=1$ in (4.1) must lack a time-reference. And, as we saw in sec.3.3, this in turn can be shown to be incompatible with QM itself.

\subsection{Van Fraassen's account}

A detailed discussion of QM measurement is presented in van Fraassen (1991).While this discussion is critical of von Neumann's classical account, it is in one sense similar to the latter. Both are part of a school of thought that distributes state plus probability and eventual outcome to different times in order to make sense of QM measurement in light of COMP. Moreover, van Fraassen also respects the value assumption. I quote a longer passage, where he characterizes QM measurement before differentiating collapse and nocollapse (modal) interpretations (one version of which he advocates). Van Fraassen writes:

Born's Rule says [... that the] probability that a measurement of yes-no observable $\mathbf{P}$ will yield value 1 , if made in state $W$, equals $\operatorname{Tr}(\mathrm{W} \mathbf{P})$. This statement gives us the probability of the occurrence of an event, conditional on the occurrence of a certain measurement process in a given state. [...] But what is this event? During the measurement process we see an initial situation change into a final situation. The initial situation can be analysed into two parts: the system of interest is in state $\mathrm{W}$, and the environment (presence of and interaction with the measuring apparatus in its groundstate) has a certain character - call it IN. In the final situation, this system's state will have evolved into, say, $\mathrm{W}^{\prime}$, and the environment will have a new character - call it OUT - which includes e.g. that the pointer on the apparatus now sits at the number 1 . So we have the transition: IN, $\mathrm{W} \rightarrow \mathrm{W}^{\prime}$, OUT. This transition could be indeterministic in two ways. Recall that we have a probability for OUT. Another possible transition - one that could have occurred but did not - may be described as IN, W $\rightarrow \mathrm{W}^{\prime \prime}$, OUT' and it too had a probability. (1991, 244-5; my italics)

The two ways of indeterminism then differentiate two types of interpretation. Either, in a no-collapse interpretation, given 'the initial situation (IN, $W$ ), the final state $W$ ' is completely determined (so $W^{\prime \prime}=W^{\prime}$ ), and the probability we are given is really the probability of character OUT, given that the system is then in state $W^{\prime}$. ' Or, in a collapse interpretation, given 'the final state, the outcome character is completely determined (OUT $\neq$ OUT' then $\left.\mathrm{W}^{\prime} \neq \mathrm{W}^{\prime \prime}\right)$, and the probability we are given is the probability of the transition from state $\mathrm{W}$ to $W^{\prime}$, when the initial situation has character IN.' $(1991,245)$.

From this passage, we learn two things about QM measurement. First, although van Fraassen does not explicate time-references for $W$ and $W^{\prime}$ (or $\left.W^{\prime \prime}\right)$ it is entirely obvious from (the italicised portions of) the text that $\mathrm{W}^{\prime}$ (or $\mathrm{W}^{\prime \prime}$ ) is the system's state at a time later than the one of W. Second, it is also clear from the text that the Born Rule probability is one for an event at the later time: $W^{\prime}$ and character OuT are both part of the 'final situation' and the original probability is either one for OUT only or for OUT plus $W^{\prime}$. All in all, van Fraassen straightforwardly explains that in the trace formula $\mathrm{p}([\mathbf{P}]=1)=\operatorname{Tr}(\mathrm{W} \mathbf{P})$ the elements ' $\mathrm{W}^{\prime}$ (state) and ' $[\mathbf{P}]=1^{\prime}$ (value) refer to different times. In a collapse interpretation, this 
probability simultaneously is one for $W^{\prime}=\mathbf{P}$ such that we can rewrite the formula as $\mathrm{p}(\mathbf{P})=\operatorname{Tr}(\mathrm{W} \mathbf{P})$. This of course implies the weaker formula. Moreover, since by assumption $\mathrm{W}^{\prime}$ is a state later than $\mathrm{W}$, in this rewritten version the two time references are even more obvious.

Explicitly, van Fraassen starts with $W$, the initial state of $S$ ('the system of interest') as an input for the trace formula. Hence, he proposes a version of formula $\mathrm{p}\left(\left[\mathbf{P}_{a k}\right]=1\right)=\operatorname{Tr}(\mathrm{W}$ $\mathbf{P}_{a k}$ ), where ' $W$ ' (initial state of $S$ ) and ' $\left[\mathbf{P}_{a k}\right]=1$ ' (value of $S$ ) refer to different times. This is a literal interpretation of the passage and I emphasize right away that it is doubtful whether it really catches van Fraassen's intention but leave that worry aside for a moment. What van Fraassen clearly does endorse is the value assumption. When expounding his own interpretation he takes the Born Rule to deliver probabilities for 'value states' or better for the truth of 'value attributing propositions' where such a proposition 'says that observable $\boldsymbol{A}$ actually has a value $\boldsymbol{a}_{\boldsymbol{k}}$ ' (van Fraassen 1991, 275; my italics and notation).

Assume now that in van Fraassen's equation p $\left(\left[\mathbf{P}_{a k}\right]=1\right)=\operatorname{Tr}\left(W \mathbf{P}_{a k}\right)$ the state $\mathrm{W}$ equals $W\left(t_{1}\right)$ where $t_{1}$ is the time of the onset of interaction between $S$ and $M$. This is clearly suggested by his characterizing $\mathrm{W}$ as the 'initial state'. Assume that the second half of my interpretation is also correct, i.e. that ${ }^{\prime}\left[\mathbf{P}_{a k}\right]=1$ ' is an equivalent of his value attributing proposition which, if true, is true at a later time. Van Fraassen then is effectively putting forward this version of the trace formula:

$$
\mathrm{p}\left(\left[\mathbf{P}_{a k}\right]=1 \text { at } \mathrm{t}^{\prime}\right)=\operatorname{Tr}\left(\mathrm{W}(\mathrm{t}) \mathbf{P}_{a k}\right)
$$

where $t^{\prime}>t$. But of course, the problem with (4.2) is the same as with von Neumann's (4.1). $\mathrm{QM}$ contains no theory giving us $\mathrm{t}^{\prime}$ for a given $\mathrm{t}$, so (4.2) cannot be a formula of QM. This means that also in van Fraassen's version of the trace formula the internal time-reference must be cancelled as a matter of principle - which in sec. 3.3 was shown to be in conflict with QM.

I have recorded doubts whether van Fraassen should be saddled with the interpretation leading to (4.2). ${ }^{18}$ Indeed, it is very clear that his account of measurement aims at another state. To quote him again, in a no-collapse interpretation the trace formula provides 'the probability of character OUT, given that the system is then in state $W^{\prime}$. ' We may read this as saying that really $W^{\prime}\left(t_{2}\right)$ of $S$ is the appropriate input for the formula. This reading is corroborated by looking at van Fraassen's own version of a no-collapse interpretation. Referring back to my discussion of a superposition measurement in sec.4.1, the interpretation holds that the state of composite system $S+M$ at $t_{2}$ equals $P_{B^{\prime}}\left(t_{2}\right)=$ $\left|\mathrm{B}^{\prime}><\mathrm{B}^{\prime}\right|$ with $\left|\mathrm{B}^{\prime}\right\rangle=1 / \sqrt{2}\left(\left|\boldsymbol{a}_{1}\right\rangle\left|\boldsymbol{m}_{1}\right\rangle+\left|\boldsymbol{a}_{2}\right\rangle\left|\boldsymbol{m}_{2}\right\rangle\right)$. At $\mathrm{t}_{2}, \mathrm{~S}$ and $\mathrm{M}$ have become correlated, their reduced states are mixtures and denial of EE allows us to ascribe values to

${ }^{18}$ I here refer to van Fraassen (1991). In an earlier exposition of his modal interpretation van Fraassen formulates an equivalent of (4.2) explicitly. He gives this version of the Born Rule: 'When $A$ is an observable pertaining to $S$ and the process w $\left(\mathrm{t}_{1}\right) \rightarrow \mathrm{w}\left(\mathrm{t}_{2}\right)$ is a measurement of $A$ on $S$ by $\mathrm{M}$, with pointer observable $M$, then $\left.\alpha_{1}\left(\mathrm{t}_{1}\right)(<A, \mathrm{E}\rangle\right)$ is the probability that $\mathrm{w}\left(\mathrm{t}_{2}\right)$ is in $\langle A, \mathrm{E}\rangle$ and equally the probability that it is in $\langle M, E\rangle$ ' (van Fraassen 1981, 305; notation adapted). Van Fraassen's 'S is in $\langle A, E\rangle$ ' is equivalent to ' $[A] \in \mathrm{E}^{\prime}$ and for discrete $A$ may be simplified to ' $[A]=\boldsymbol{a}_{k}$ ' or equivalently ' $\left[\mathrm{P}_{a k}\right]=1^{\prime}$. $\alpha$ is a map from the set of value attributions into [0,1] given by $\alpha(\langle A, E\rangle)=\operatorname{Tr}\left(\alpha \mathbf{P}_{M, E}\right)$, where $\alpha$ is a density operator, i.e. in the present notation we can introduce a similar map W with: $W\left(\left[\mathbf{P}_{a k}\right]=1\right)=\operatorname{Tr}\left(W \mathbf{P}_{a k}\right)$. Putting in time references from the quotation, we get $\mathrm{p}\left(\left[\mathbf{P}_{a k}\right]=1\right.$ at $\left.\mathrm{t}_{2}\right)=\operatorname{Tr}\left(\mathrm{W}\left(\mathrm{t}_{1}\right) \mathbf{P}_{a k}\right)$ - which is an instance of (4.2). 
observables $\boldsymbol{A}$ and $\boldsymbol{M}$ (on $\mathrm{S}$ and $\mathrm{M}$ ). This again seems to imply that it is a state $\mathrm{W}^{\prime}\left(\mathrm{t}_{2}\right)$ (e.g. a reduced state of $S$ ) that should go into the trace formula - at least in a no-collapse interpretation. (Note that for a collapse interpretation using $\mathrm{W}^{\prime}\left(\mathrm{t}_{2}\right)$, a collapsed state as an input for the formula would not, generally make sense.)

Van Fraassen's exposition only suggests this alternative interpretation but another interpreter, Vermaas $(1999,195)$, explicates it - for a certain type of state $W^{\prime}\left(t_{2}\right) .{ }^{19}$ Vermaas also endorses a no-collapse interpretation and is motivated by a similar account of measurement. He uses $W^{\prime}\left(t_{2}\right)$, where this is the reduced state of the apparatus $M$, in order to calculate probabilities for values of the pointer observable $M$ or explicitly: $\mathrm{p}\left([\mathbf{P}]=1\right.$ at $\left.\mathrm{t}_{2}\right)=$ $\operatorname{Tr}\left(\mathrm{W}^{\prime}\left(\mathrm{t}_{2}\right) \mathbf{P}\right)$ (where $\mathbf{P}$ now is a projector onto an eigenspace of $\left.\mathbf{M}\right){ }^{20}$ Apparently, it seems unobjectionable in this particular situation to write the trace formula like this. But of course the formula cannot plausibly be restricted to this situation. We cannot help ourselves to a version here which we are not obliged to in general. When we consider possible interpretations of the trace formula any restriction to a certain type of state (apparatus mixture) is illegitimate. Neither the formula nor any standard version of the Born Rule (where it ultimately resides) nor the algorithm for expectation values know of any such restriction. And it is this fact that has led me to explicitly claim Generality of the trace formula as a property of QM. Now, if we read Vermaas' version as a general proposal for interpreting the trace formula then clearly we are back to square one. We must allow $W\left(t_{1}\right)$ as a possible input for the formula, where $W\left(t_{1}\right)$ is $S^{\prime}$ s state at an arbitrary time (it might be measurement onset or not). According to the proposal Vermaas makes (using $W^{\prime}\left(t_{2}\right)$ !), we then have, for (the equally admissible) $W\left(t_{1}\right)$, the formula $\mathrm{p}\left(\left[\mathbf{P}_{a k}\right]=1\right.$ at $\left.\mathrm{t}_{1}\right)=\operatorname{Tr}\left(\mathrm{W}\left(\mathrm{t}_{1}\right) \mathbf{P}_{a k}\right)$. But this just is the internal interpretation of the formula from sec.3.2. If $\mathrm{W}\left(\mathrm{t}_{1}\right)$ is a superposition of $A$-eigenstates this interpretation is straightforwardly brought into conflict with COMP.

One may question whether I am doing justice to the proposal. What is intended is a version of the trace formula: $\mathrm{p}\left([\mathbf{P}]=1\right.$ at $\left.t_{2}\right)=\operatorname{Tr}\left(\mathrm{W}^{\prime}\left(\mathrm{t}_{2}\right) \mathbf{P}\right)$, where $\mathrm{W}^{\prime}\left(\mathrm{t}_{2}\right)$ is an arbitrary state in the sense of pertaining either to $S, M$ or $S+M$, but a non-arbitrary one in the sense of referring to a time $t_{2}$ after measurement onset. By contrast, $W\left(t_{1}\right)$ does not unambiguously refer to such a time, indeed can be taken to be the state at measurement onset. So the idea is to formulate a version of the trace formula roughly like this:

$$
\mathrm{p}\left(\left[\mathbf{P}_{a k}\right]=1 \text { at } \mathrm{t}_{2}\right)=\operatorname{Tr}\left(\mathrm{W}^{\prime}\left(\mathrm{t}_{2}\right) \mathbf{P}_{a k}\right)
$$

where $W^{\prime}\left(t_{2}\right)$ explicitly is considered as a state after measurement onset. Taking $t_{1}$ to be measurement onset, $\mathrm{W}\left(\mathrm{t}_{1}\right)$ is not a legitimate input for (4.2). I understand the idea but still cannot see how it respects the QM property of Generality. At an arbitrary time $t$, the system's state is represented by an arbitrary member $\mathrm{W}(\mathrm{t})$ of the set of all possible density operators on the appropriate space and the trace formula processes that state. So to respect Generality, W $\left(t_{1}\right)$ has to be an admissible input.

One may propose that Generality should be relinquished. I have two replies. First of all, I insist that Generality is a feature of ordinary QM. No version of the trace formula known to me makes any restrictions on the allowed input states and observables. ${ }^{21} \mathrm{KS}$ type theorems

\footnotetext{
19 Vermaas here characterizes a common property of three modal interpretations that are all stronger than van Fraassen's (see Vermaas 1999, ch.4), so we may take his proposal as an interpretation of the trace formula in modal interpretations, in general.

${ }^{20}$ This explicitly is the content of Vermaas' proposal $(1999,195)$.

${ }^{21}$ In particular, this is true for the references in note 9 above.
} 
concern observables in ordinary QM, COMP was intended as an interpretation of these theorems referring to ordinary $\mathrm{QM}$, and it is ordinary QM that has been shown to be in a conflict with COMP. So the proposal to relinquish Generality amounts to a change of the subject because it does not talk about ordinary QM.

Second, I do not see how (4.3) really could work. Since Generality is a formal property of $\mathrm{QM}$, to abandon it means to substantially revise the formalism and it is not a priori clear whether the revision will give us a sensible new QM. Consider this. Regardless of whether we interpret $\mathrm{W}^{\prime}\left(\mathrm{t}_{2}\right)$ in (4.3) as a state of $\mathrm{S}, \mathrm{M}$ or $\mathrm{S}+\mathrm{M}$, it is a state at some time during (or at the end of) an $\boldsymbol{A}$-measurement, hence is not independent of a choice of observable $\boldsymbol{A}$. Accordingly, the trace formula is no longer general in yet another sense, i.e. it no longer processes arbitrary observables. Suppose that we want to calculate $\mathrm{W}^{\prime}\left(\mathrm{t}_{2}\right)$ via the QM timeevolution directly from the preparation $\mathrm{W}\left(\mathrm{t}_{0}\right)$ - insisting with (4.2) that an $A$-measurement must start at some time $t_{1}$ in between, but not specifying a state $W\left(t_{1}\right)$ - then $W\left(t_{0}\right)$ has to include some state of the $A$-measurement apparatus leading to the appropriate 'ready'-state, i.e. we would no longer be able to refer to states of QM systems apart from devices measuring specific observables on them. Suppose alternatively that we want to recover something like Generality, given (4.3). Assume that, for observables $\boldsymbol{A}$ and $\boldsymbol{B}$ with $[\boldsymbol{A}, \boldsymbol{B}] \neq 0$, we want the trace formula to yield probabilities for both choices. This inevitably will force that we do explicate a state $\mathrm{W}\left(\mathrm{t}_{1}\right)$ of $\mathrm{S}$ alone (calculated from $\mathrm{W}\left(\mathrm{t}_{0}\right)$ and with $\left.\mathrm{t}_{0}<\mathrm{t}_{1}<\mathrm{t}_{2}\right)$ that is neutral between those choices. So the algorithm containing the new trace formula (4.3) (a new version of the Born Rule) will have to make reference to two different states $W\left(t_{1}\right)$ and $W^{\prime}\left(t_{2}\right)$. And this will have to involve an exact theory about how the two states are related, i.e. given $W\left(t_{1}\right)$, where $t_{1}$ may be measurement onset, we must name sufficient conditions for how to pick time $t_{2}$ such that $W^{\prime}\left(t_{2}\right)$ can be put into the trace formula. The usual choice - that $t_{2}$ is a time when $S$ and $M$ have become perfectly correlated (see van Fraassen 1991, 200) - is unavailable because that correlation presupposes an apparatus measuring a specific observable, mention of which we just wanted to avoid.

In sum, (4.3) simply does not respect one of the properties of QM, i.e. Generality. Giving up this property forces a formidable revision of QM that does not yet exist. I don't think that modal interpreters like van Fraassen or Vermaas really intend such a revision. But the only alternative to (4.3) is the literal interpretation of van Fraassen's general account of measurement - in effect (4.2). The argument of sec.3.2 can be viewed as explaining why interpreters put forward proposals like this. (Van Fraassen, though exceptionally clear on the matter, certainly is not the only interpreter doing so; vide von Neumann.) And sec.3.3 above presents an argument why (4.2), like (4.1) before, cannot be a component of QM. All in all, both van Fraassen and von Neumann exemplify an important tradition of thought about QM measurement that as a whole is challenged by the above argument. Our consideration of this tradition, rather than showing how to avoid or mitigate the original completeness problem, exposes it even more starkly.

\section{Objection}

The mentioned school of thought about QM measurement tries to accommodate COMP by accepting the value assumption and suggesting two time references, either by separating the time references of state and eventual value ((4.1 and (4.2)) or by tacitly referring to a state at a time after measurement onset ((4.3)). Both roads, I have argued, are blocked. So we should now consider the remaining alternative. Could the value assumption be dropped? 
This is a fundamental objection and it makes the whole argument of sec.3.2 collapse immediately. To repeat: Given the value assumption, in the trace formula $\mathrm{p}\left(\boldsymbol{a}_{k}\right)=\mathrm{p}\left([A]=\boldsymbol{a}_{k}\right)$ where ' $[A]=\boldsymbol{a}_{\boldsymbol{k}}{ }^{\prime}$ means that $\mathrm{S}$ has value $\boldsymbol{a}_{\boldsymbol{k}}$ of $A$. Now, choose $\mathrm{W}(\mathrm{t})$ such that $\mathrm{p}\left(\boldsymbol{a}_{\boldsymbol{k}}\right)>0$ and, by COMP, $\neg[A]=\boldsymbol{a}_{k}$ at $\mathrm{t}$. Setting $\mathrm{p}\left(\boldsymbol{a}_{k}\right)=\mathrm{p}\left(\boldsymbol{a}_{\boldsymbol{k}}(\mathrm{t})\right)$ we get an implausibility and, assuming the probability principle, a contradiction. It all depends on the first step: the value assumption. To drop it means to assume that QM probabilities do not concern events that can be identified with $S$ having a value (of an observable) at a specific time. Instead they concern events of another type - but events that can be assumed to occur at time $t$, where $t$ is the parameter from $W(t)$.

We can very broadly delimit what these events are. The purpose of giving up the value assumption is to find a replacement for ${ }^{\prime}[A]=a_{k}$ at $\mathrm{t}^{\prime}$ in $\mathrm{p}\left([A]=\boldsymbol{a}_{k}\right.$ at $\left.\mathrm{t}\right)$ such that the latter does not (via the probability principle) get into conflict with $\neg[A]=a_{k}$ at $\mathrm{t}$, from COMP (given a pure non-eigenstate of $A$ ). A positive suggestion for the events is that they are situations which are as if $[A]=\boldsymbol{a}_{k}$ at $\mathrm{t}$. Let's write $\mathrm{p}\left(\right.$ “ $[A]=\boldsymbol{a}_{k}$ at $\left.\mathrm{t}^{\prime \prime}\right)$ - using double quotes for the probability that the situation at $\mathrm{t}$ is as if $S$ had $\boldsymbol{a}_{k}$ at $\mathrm{t}$. In fact, this is the broadest construal of an alternative to $\mathrm{p}\left([A]=\boldsymbol{a}_{k}\right.$ at $\left.\mathrm{t}\right)$ - no double quotes - that I can think of. Its appeal, I believe, comes from the following intuition. A physical situation can have features that are as if some fact $\mathrm{F}$ were the case while in fact it must, for whatever reason, remain open whether really $\mathrm{F}$ is the case.22 Think, e.g., of a concrete reading of a value of pointer observable $\boldsymbol{M}$ from which we draw conclusions to $S$ values (perhaps at the same or perhaps at an earlier time). Isn't the whole situation at the end of a measurement as if $\mathrm{S}$ had $\boldsymbol{a}_{\boldsymbol{k}}$ (at some time, leave that open for the moment)? And isn't it plausible that one can remain agnostic whether $S$ really had $\boldsymbol{a}_{k}$ (at that time)?

This interpretation of the 'as if'-proposal may be appealing but here it is not available. Recall once more the structure of the argument in sec.3.2 and plug in the 'as if'-proposal. Choose $\mathrm{W}(\mathrm{t})$ such that $\mathrm{p}\left(\right.$ " $[A]=\boldsymbol{a}_{k}$ at $\left.\mathrm{t}^{\prime \prime}\right)>0$ and, by COMP, $\neg[A]=\boldsymbol{a}_{k}$ at $\mathrm{t}$. Since the probability is positive the probability principle applies, i.e. the proposition "within" the probability must be consistent with $\neg[A]=a_{k}$ at t. For a check, resolve the double quotes of that proposition in the manner suggested: 'It is as if $[A]=\boldsymbol{a}_{k}$ at $\mathrm{t}$ but in fact it remains open whether $[A]=\boldsymbol{a}_{k}$ at $\mathrm{t}^{\prime}$. Clearly this sentence does not safely avoid a contradiction with ' $\neg[A]=a_{k}$ at $\mathrm{t}^{\prime}$. So the appealing version of the 'as if'-interpretation is not the one that can salvage the consistency of complete QM. Instead what we need is that propositions of type " $[A]=a_{k}$ at $\mathrm{t}^{\prime \prime}$ (with double quotes) are interpreted as follows: 'It is as if $[A]=a_{k}$ at $\mathrm{t}$ but in fact it is not the case that $[A]=\boldsymbol{a}_{\boldsymbol{k}}$ at $\mathrm{t}^{\prime}$. This statement is the only explication safely consistent with ' $\neg[A]=a_{k}$ at $\mathrm{t}^{\prime}$ and hence is the only available reading of the 'as if'-interpretation. But now recall that the proposal must be taken as an alternative reading of all probabilities within the trace formula. So, if we adopt the 'as if'-proposal we automatically propose what QM probabilities are, in general. Such probabilities now concern the mere display of values. (For contrast, recall the display of number 6 in the die in sec.4.2). Writing the display of a value

\footnotetext{
22 This also is a plausible interpretation of von Neumann's idea that in a QM context experience only consists in statements of '(subjective) observation', not statements ascertaining what is the case (see note 17). I have argued above that in the context of his 'taking on'-idea probabilities must concern what is the case, hence the value assumption must be respected. Here, I explore the idea that probabilities do concern only what we experience, not what is the case, i.e. that the value assumption is rejected. I do not think that von Neumann can coherently maintain both his ideas but I will not try to show this as it would embroil me in questions of text exegesis.
} 
in French quotes, we have that “ $a_{i}$ " $\leftrightarrow « a_{i} » \& \neg \boldsymbol{a}_{i}$. We have that an 'as if'-probability p (" $\left.a_{i}^{\prime \prime}\right)$ equals p (« $\boldsymbol{a}_{i} » \& \neg \boldsymbol{a}_{i}$ ) (for all i).

As an interpretation of a fundamental physical theory this sounds wildly implausible and it can indeed be shown to be so. Consider what can be the only motivation for the proposal: to harmonize QM and completeness in the form of either EE or COMP. So the proposal had better be compatible with these two assumptions. But it cannot be so if it also respects Generality, i.e. is a state-independent version of the trace formula. Assume that the formula is now to be read as:

$$
\mathrm{p}\left(“[A]=a_{k} \text { at } \mathrm{t}^{\prime \prime}\right)=\operatorname{Tr}\left(\mathrm{W}(\mathrm{t}) \mathbf{P}_{a k}\right)
$$

for arbitrary $\mathrm{W}(\mathrm{t})$ and $A$. Now, suppose that $\mathrm{W}(\mathrm{t})=\mathbf{P}_{a k}$. Then, from (5.1) $\mathrm{p}\left({ }^{\prime \prime} a_{k}(\mathrm{t}) "\right)=1$. But this equals the following probability: $\mathrm{p}\left(" \boldsymbol{a}_{k}(\mathrm{t}) » \& \neg \boldsymbol{a}_{k}(\mathrm{t})\right)$. Since the probability of this conjunction equals 1 we may assume that both conjuncts are true. In particular it is true that $\neg[A]=\boldsymbol{a}_{k}$ at $\mathrm{t}$. We have deduced: If $\mathrm{S}$ is in $\mathrm{W}(\mathrm{t})=\mathbf{P}_{a k}$ then $\neg[A]=\boldsymbol{a}_{k}$ at $t$. This contradicts EE, so a defender of full EE cannot maintain (5.1) for all states.

Given COMP, there is no contradiction but still we have deduced that if $\mathrm{S}$ is in $\mathrm{W}(\mathrm{t})=\mathbf{P}_{a k}$ then $\neg[A]=a_{k}$ at $\mathrm{t}$. Since $A$ is arbitrary and $\mathrm{k}$ is an arbitrary one of the indices $\mathrm{i}$, we have derived that if $S$ is in an arbitrary $A$-eigenstate then it does not have a value of $A$. But recalling COMP, when $S$ is in an $\boldsymbol{A}$-eigenstate then it does not have a value of $\boldsymbol{B}$, for any $\boldsymbol{B}$ with $[A, B] \neq 0$. So when $S$ is in an arbitrary pure state, it does not have a value of any observable. This is an implausible result. We should recall why COMP was endorsed in the first place, namely to accommodate the classical no hidden variables proofs (of KS-type). These proofs show that (given two assumptions) observables on $\mathrm{S}$ forming a KS set cannot all be assumed to simultaneously have values. Now, we learn that given S's state is pure, none of the observables in an arbitrary set has a value anyway (at a given time). So, given a pure state the claims of all KS-type proofs are vacuously true. Now, if S's state is an ignorance mixture then $S$ really is in one of the pure states we happen to be ignorant about, so assuming $S^{\prime}$ s state to be such a mixture will not make a difference. So the defender of (5.1) and COMP must claim that only if $S$ is in a mixture not interpretable as an expression of our ignorance, i.e. a reduction mixture, it can be assumed to have a value of some observable and the KS arguments are non-vacuously true. However, it is perfectly admissible to assume S say, Kochen and Specker's original spin-1 system - to be the only QM system in the universe. In this case, there is no larger system such that S's reduced state could be interpreted as a non-trivial reduction mixture - which again means that the KS arguments are only vacuously true. Hence, this line of reasoning is implausible. Accordingly, the 'as if'interpretation cannot deliver a version of the trace formula that is plausible and respects Generality.

As in the case of proposal (4.3), one may seriously consider giving up Generality. Again, this means to give up something that is a property of ordinary QM. Abandoning it may be a possible reaction to the argument (which concerns ordinary QM) but certainly is no valid objection. And again the price of abandoning Generality is high. It is a formal property of $\mathrm{QM}$, hence without it we must revise the formalism. Neither has such a revision been tried anywhere nor is it obvious how a reasonable new QM could arise. Suppose that we bite the bullet and dismiss the project of one general trace formula. We would have two trace formulas instead: 


$$
\begin{gathered}
\mathrm{p}\left(“[A]=a_{k} \text { at } \mathrm{t}^{\prime \prime}\right)=\operatorname{Tr}\left(\mathrm{W}(\mathrm{t}) \mathbf{P}_{a k}\right), \quad \text { if } \mathrm{W}(\mathrm{t}) \neq \mathbf{P}_{a i} \text {, for any i; } \mathrm{W}(\mathrm{t}) \text { pure } \\
\mathrm{p}\left([A]=\boldsymbol{a}_{k} \text { at } \mathrm{t}\right)=\operatorname{Tr}\left(\mathrm{W}(\mathrm{t}) \mathbf{P}_{a k}\right) \quad \text { if } \quad \mathrm{W}(\mathrm{t})=\mathbf{P}_{a i} \text {, for any i }
\end{gathered}
$$

According to (5.2b), for the eigenstates of $A$ we have $\mathrm{p}\left(\boldsymbol{a}_{k}(\mathrm{t})\right)=\delta_{i k}$ for $\mathrm{W}(\mathrm{t})=\mathbf{P}_{a i}$. And by (5.2a), p (" $a_{k}(\mathrm{t})$ ") is the appropriate probability for a pure non-eigenstate of $A$. How to treat mixed states? Assume first that they are lumped together with the $\mathbf{P}_{a i}$ in (5.2b), i.e. the trace formula produces probabilities for the truly possessed values for these mixtures (rather then the 'as if'-probabilities of (5.2a)). It seems plausible that for an observable $\boldsymbol{A} \otimes \boldsymbol{B}$ on a compound system the 'as if'-interpretation (5.2a) is true with respect to $A \otimes B$ if and only if it is true for $A$ and $B$ Now, let $\mathrm{S}+\mathrm{M}$ be in state $\mathbf{P}_{B}=|\mathrm{B}\rangle\langle\mathrm{B}|$ with $\left.|\mathrm{B}>=1 / 2(\sqrt{ } 3)| \boldsymbol{a}_{1}\right\rangle\left|\boldsymbol{m}_{1}\right\rangle+1 / 2\left|\boldsymbol{a}_{2}\right\rangle\left|\boldsymbol{m}_{2}\right\rangle$, where the $\left|\boldsymbol{a}_{i}\right\rangle$ and $\left|\boldsymbol{m}_{i}\right\rangle($ for $\mathrm{i}=1,2)$ are eigenstates of $\boldsymbol{A}$ and $\boldsymbol{M}$, respectively. Then the reduced states $\mathbf{P}_{B} \#$ of $S$ and $\# \mathbf{P}_{B}$ of $\mathrm{M}$ are mixtures of the respective eigenstates and the probabilities are calculated via $(5.2 b)$, according to our assumption. But $S+M$ is in a pure non-eigenstate of $\boldsymbol{A}+\boldsymbol{M}$, hence the 'as if'-version (5.2a) applies. Since e.g. $\operatorname{Tr}\left(\mathbf{P}_{m 1}\left(\# \mathbf{P}_{B}\right)\right)=\operatorname{Tr}\left(\left(\mathbf{P}_{a 1} \otimes \mathbf{P}_{m 1}\right) \mathbf{P}_{B}\right)$ (where $\mathbf{P}_{a 1}$ and $\mathbf{P}_{m 1}$ project onto rays containing $\left|\boldsymbol{a}_{1}\right\rangle$ and $\left|\boldsymbol{m}_{1}\right\rangle$, respectively, in the respective subspaces) we have that $\mathrm{p}\left(\boldsymbol{m}_{1}\right)=\mathrm{p}\left(" \boldsymbol{a}_{1}\right.$ " \& " $m_{1}$ ") and since $\mathbf{P}_{B}$ is a perfectly correlated state also $\mathrm{p}\left(\boldsymbol{m}_{1}\right)=$ $\mathrm{p}\left(\right.$ ( $m_{1}$ "). Now, events « $m_{i} » \& \neg m_{i}$ are also perfectly correlated (for otherwise we blur the distinction between (5.2a and 5.2b)). Hence, $\mathrm{p}\left(\boldsymbol{m}_{1}\right)=\mathrm{p}\left(“ \boldsymbol{m}_{1}\right.$ ") $=\mathrm{p}\left(« \boldsymbol{m}_{1} » \& \neg \boldsymbol{m}_{1}\right)=\mathrm{p}\left(\neg \boldsymbol{m}_{1}\right)$. This means that $\mathrm{p}\left(\boldsymbol{m}_{1}\right)=\mathrm{p}\left(\neg \boldsymbol{m}_{1}\right)=3 / 4$. Hence, we cannot plausibly treat mixtures via (5.2a). Suppose alternatively that we treat mixtures like pure non-eigenstates of $A$, i.e. apply an 'as if'-interpretation as in (5.2a). Assume that the system in question is the measurement apparatus $M$, that there is an observable $M$ on $M$ and that the state of $S+M$ is a superposition such that M's reduced state \# $\mathbf{P}\left(\mathrm{t}_{1}\right)=\Sigma_{\mathrm{i}} \mathrm{a}_{\mathrm{i}} \mathbf{P}_{m i}$, where the $\mathbf{P}_{m i}$ project onto the pointer eigenstates. Then for this state (of M!) the conditions for (5.2a) are in place. Hence, we have probabilities $\mathrm{p}\left({ }^{\prime \prime} \boldsymbol{m}_{i}{ }^{\prime}\right)=\mathrm{p}\left(\right.$ « $\left.\boldsymbol{m}_{i} » \& \neg \boldsymbol{m}_{i}\right)$ (for all i), i.e. each probability concerns the event that the $M$-measurement apparatus $M$ is in a situation that is as if $\mathrm{M}$ had $\boldsymbol{m}_{i}$ at $\mathrm{t}_{1}$ and $M$ does not have $m_{i}$ at $t_{1}$ (for all i). But $M$ is an observable accessible to the naked eye. So QM plus $(5.2 \mathrm{a}, \mathrm{b})$ proposes that direct observation of a macroscopic object is regularly mistaken and universally so, if we have reason to believe the observed object $\mathrm{M}$ is in a non-eigenstate of the pointer observable.

We need to hear nothing further, I believe. An empirical theory that must assume systematic error in the direct observations eventually testing it is absurd. So the 'as if'-proposal fails not only as a general proposal for the trace formula, but also in terms of its content. ${ }^{23}$ Hence, the only tangible proposal not obeying the value assumption fails. The argument of sec.3 remains intact.

\footnotetext{
${ }^{23}$ Note also that the measurement problem, in the second version above, is trivially dissolved. Nothing prohibits that we calculate probabilities for the values of pointer observables (of a macroscopic apparatus) from the trace formula. But these must now, of course, also be probabilities for 'as if'-events. Consider the probability $\mathrm{p}\left(\right.$ " $[M]=m_{1}$ at $\mathrm{t}_{3}$ ") deduced from a state $\mathrm{W}\left(\mathrm{t}_{3}\right)$ of $\mathrm{S}+\mathrm{M}$ and $\mathrm{t}_{3}$ is the end of the $\mathrm{S}-\mathrm{M}$ interaction. We have a probability for a situation that is as if $\mathrm{M}$ showed $m_{1}$ at $\mathrm{t}_{3}$ while $\mathrm{M}$ does not in fact show $m_{1}$ at $t_{3}$. If we take to heart this interpretation then there can of course be no measurement problem. This problem was that we see, e.g., $[M]=m_{1}$ at $t_{3}$ while the superposition affecting $M$ prohibits (via EE) that $[M]=m_{1}$ at $t_{3}$. But given the 'as if'-interpretation our vision is deceptive anyway. It is not the case that $[M]=m_{1}$ at $t_{3}$. If our observations are by assumption fallacious they can be in conflict with ascribed QM states but the conflict is harmless just because they are fallacious. Cancelling one of two contradictory statements always removes a contradiction.
} 


\section{Consequences}

The argument here developed shows that EE, the standard interpretation of QM completeness originating with Dirac and von Neumann, is implausible. Given State Uniqueness, EE entails COMP, which cannot be true under plausible assumptions. This may seem but a minor result. While many theoreticians not concerned with the foundations of QM still reproduce EE many others concerned with these foundations reject it right away. But the above result is of considerable strength. First of all, adopting or rejecting EE in the past often was a matter of general interpretive preferences, so any argument relating it to the structure of QM itself must be welcome. Moreover, in the presence of State Uniqueness, which is a property of QM anyway, COMP is weaker than EE, hence the result is stronger. Finally, the assumptions made are mostly very general and so the argument should guide our future thought about time-references in the formalism of QM.

Of course, if the result is any good then its trivial consequence is that, given the assumptions, the negation of COMP is true. But the discussion hopefully has highlighted what exactly this negation is: If $S$ is in some state $W(t)$ such that for an arbitrary observable $\mathbf{P}_{a k}$ the trace formula yields: $1>\mathrm{p}\left(\boldsymbol{a}_{k}\right)=\operatorname{Tr}\left(\mathrm{W}(\mathrm{t}) \mathbf{P}_{a k}\right)>0$ (i.e. $\mathrm{W}(\mathrm{t})$ is a non-eigenstate of $\left.\boldsymbol{A}\right)$, then this must be explicated by the internal interpretation, hence must explicitly run: $\mathrm{p}\left(\boldsymbol{a}_{k}(\mathrm{t})\right)>0$ and it must be legitimate to assume that $\mathrm{S}$ has value $\boldsymbol{a}_{k}$ of $\boldsymbol{A}$ at time $\mathrm{t}-$ even when $W(t)$ is a pure non-eigenstate of $\boldsymbol{A}$. It should be obvious that this conclusion, if inescapable, has radical implications. Let me nevertheless illustrate them by means of a well-known and elegant KS variant due to Mermin (1990). Let $S$ be a two-particle spin $1 / 2$ system (hence we must have a space $\mathcal{H}$ with $\operatorname{dim} \mathcal{H}=4$ ) and consider (for an orthogonal triple $\mathrm{x}, \mathrm{y}, \mathrm{z}$ of vectors in $R^{3}$ ) these nine spin observables: $\sigma^{1}{ }_{x}, \sigma^{2} x, \sigma^{1} y, \sigma_{y}^{2}, \sigma_{x}^{1} \sigma_{x}^{2}, \sigma_{y}^{1} \sigma^{2}{ }_{y}, \sigma^{1}{ }_{x} \sigma_{y}^{2}, \sigma_{y}^{1} \sigma_{x}^{2}, \sigma_{z}^{1} \sigma_{z}^{2}$. Writing again $[A]$ for the value of an observable $A$, one can derive:

\begin{tabular}{|c|c|}
\hline$\left[\sigma_{x}^{1}\right]\left[\sigma_{x}^{2}\right]\left[\sigma_{x}^{1} \sigma^{2} x\right]$ & $=$ \\
\hline$\left[\sigma_{x}^{1}\right]\left[\sigma_{y}^{2}\right]\left[\sigma_{x}^{1} \sigma_{y}^{2}\right]$ & $=$ \\
\hline$\left[\sigma_{\mathrm{y}}^{1}\right]\left[\sigma_{\mathrm{x}}^{2}\right]\left[\sigma_{\mathrm{y}}^{1} \sigma^{2} \mathrm{x}\right]$ & $=$ \\
\hline$\left[\sigma_{\mathrm{y}}^{1}\right]\left[\sigma^{2} \mathrm{y}\right]\left[\sigma_{\mathrm{y}}^{1} \sigma^{2} \mathrm{y}\right]$ & $=$ \\
\hline$\left[\sigma^{1}{ }_{x} \sigma^{2} y\left[\left[\sigma_{y}^{1} \sigma^{2} x\right]\left[\sigma_{z}^{1} \sigma_{z}^{2}\right]\right.\right.$ & $=$ \\
\hline$\left[\sigma^{1}{ }_{x} \sigma^{2} x\right]\left[\sigma^{1}{ }_{y} \sigma^{2} y\right]\left[\sigma^{1}{ }_{z} \sigma_{z}^{2}\right]$ & $=$ \\
\hline
\end{tabular}

The derivation uses the constraints mentioned in sec.2.2: Functional Composition (here the Product Rule: $[A B]=[A][B]$ ) and Noncontextuality (every operator represents a unique observable such that two occurrences of some $[A]$ have the same value). The result is so elegant because the impossibility is so easy to see. Since every value appears twice the product of all the left sides must be positive, while the one of all the right sides is negative. Now let $S$ be in the singlet state. Then it is straightforward to show that all nine observables get strictly positive probabilities. Hence, given $\neg$ COMP, it must be possible to assume that all nine observables have values at time $t$. Since this is impossible given the two constraints at least one of them must be false.

The problem outlined should direct further research in the foundations of QM. As indicated the measurement problem over which so much ink has been spilled is a consequence of yoking COMP and QM together despite the hidden inconsistency I have tried to uncover here. The only conclusion I can see is that attempts to resolve the measurement problem are misguided and that we must again investigate hidden-variables interpretations more 
seriously. It should give us pause to see that elementary textbooks of QM directed at undergraduates and ignorant of interpretational subtleties rectify, without further ado, equivalents of $\mathrm{p}\left(\boldsymbol{a}_{k}\right)=\operatorname{Tr}\left(\mathrm{W}(\mathrm{t}) \mathbf{P}_{a k}\right)$ into $\mathrm{p}\left(\boldsymbol{a}_{k}(\mathrm{t})\right)=\operatorname{Tr}\left(\mathrm{W}(\mathrm{t}) \mathbf{P}_{a k}\right)$, that theoretical physicists defining transition probabilities and propagators do so as well as those trying to wed quantum theories and general relativity while orthodox interpretations either ignore the problem or, via the measurement-outcome idea, smuggle in references to times other than $t$ that simply are not provided by $\mathrm{W}(\mathrm{t})$. To my mind, all this recommends a sincere look at how far away we are from understanding QM.

The next logical step is to try and answer which of the two conditions - Functional Composition and Noncontextuality - QM systems violate. If we look at the two possibilities of contextuality discussed in the (not so recent) literature then measurement-induced Contextuality is not an option because, as we saw, QM systems possess the properties for which we make QM predictions at measurement onset. The alternative, called Ontological Contextuality (see Redhead 1987, 137), is little more than a label and so far it is nothing that can guide an understanding of the non-Boolean lattice structure of QM observables. But here, to my mind, should be the starting point for future research. Finally, it should be pointed out that the argument relates predictions of properties of QM systems to the times of measurement onset in measurements of these systems. This does entail that the properties measured in a QM system exist in it at measurement onset, but it does not entail that measurement is faithful in the sense of revealing pre-existing properties. QM properties might very well be relational in the sense of being relations between system and apparatus or between system and observer. It is just that these properties, if they are relations, come into existence, at the latest, at the time system and apparatus meet. To make sense of QM under these conditions is certainly a fundamental task for present philosophy of physics.

\section{References}

Albert, D. (1992). Quantum mechanics and experience. Cambridge, Massachusetts: Harvard University Press.

Bub, J. (1999). Interpreting the quantum world. Cambridge: Cambridge University Press. Revised paperback edition of the 1997 edition.

Butterfield, J. (1993). Forms for probability ascriptions. International Journal of Theoretical Physics, 32, 2271-2286.

Butterfield, J. (1995). Quantum theory and the mind I: worlds, minds and quanta. Proceedings of the Aristotelian Society Supplementary Volume LXIX.

Dirac, P.A.M. (1930/1958). The principles of quantum mechanics. Oxford: Clarendon Press, 1930. $4^{\text {th }}$ edition, 1958.

Earman, J. (1986). A primer on determinism. Dordrecht: Reidel.

Fine, A. (1973). Probability and the interpretation of quantum mechanics. British Journal for the Philosophy of Science, 24, 1-37.

Greenberger, D., Hentschel, K., and Weinert, F. (eds.) Compendium of quantum physics. Berlin: Springer, 64-70.

Halpin, J.F. (1991). What is the logical form of probability assignment in quantum mechanics? Philosophy of Science, 58, 36-60.

Held, C. (2008). Axiomatic quantum mechanics and completeness. Foundations of Physics, 38, 707-732.

Held, C. (2009). The Kochen-Specker theorem. In Greenberger et al. (2009), 331-35. 
Held, C. (in preparation). Incompatibility of standard quantum completeness and quantum mechanics.

Hughes, R.I.G. (1989). The structure and interpretation of quantum mechanics. Cambridge, Mass.: Harvard University Press.

Kochen, S. \& Specker, E. (1967). The problem of hidden variables in quantum mechanics. Journal of Mathematics and Mechanics, 17, 59-87.

Landsman, K. (2009). The Born rule and its interpretation. In Greenberger et al. (2009), 64-70.

Mermin, N. D. (1990). Simple unified form of the major no-hidden variables theorems. Physical Review Letters, 65, 3373-3376.

Nielsen, M.A. \& Chuang, I.L. (2000). Quantum computation and quantum information. Cambridge: Cambridge University Press.

Redhead, M. (1987). Incompleteness, nonlocality, and realism: a prolegomenon to the philosophy of quantum mechanics. Oxford: Clarendon Press

Van Fraassen, B. C. (1981). A modal interpretation of quantum mechanics. In E. Beltrametti and B. C. van Fraassen (eds.) Current issues in quantum logic (Singapore: World Scientific): 229-58. Reprinted in L. Sklar (ed.). The philosophy of physics (New York: Garland, 2000): 279-308.

Van Fraassen, B.C. (1991). Quantum mechanics: an empiricist view. Oxford: Clarendon Press.

van Fraassen, B.C. and Hooker, C.A. (1976). A semantic analysis of Niels Bohr's philosophy of quantum theory. In W.L. Harper and C.A. Hooker (eds.): Foundations of Probability Theory, Statistical Inference, and Statistical Theories of Science, vol. III: Foundations and Philosophy of Statistical Theories in the Physical Sciences, (Dordrecht, Reidel) 221-241.

Von Neumann, J. (1932/1955). Mathematische Grundlagen der Quantenmechanik. Berlin: Springer, 1932; english translation: Mathematical foundations of quantum mechanics. Princeton: Princeton University Press, 1955. 


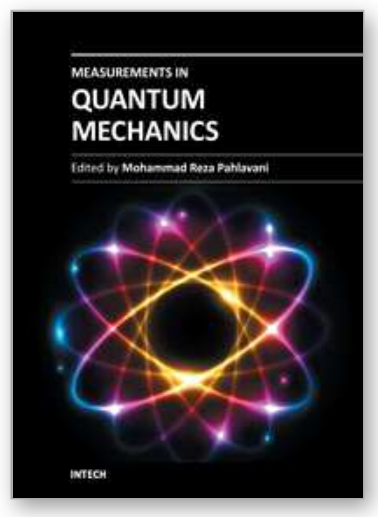

\author{
Measurements in Quantum Mechanics \\ Edited by Prof. Mohammad Reza Pahlavani
}

ISBN 978-953-51-0058-4

Hard cover, 348 pages

Publisher InTech

Published online 22, February, 2012

Published in print edition February, 2012

Perhaps quantum mechanics is viewed as the most remarkable development in 20th century physics. Each successful theory is exclusively concerned about "results of measurement". Quantum mechanics point of view is completely different from classical physics in measurement, because in microscopic world of quantum mechanics, a direct measurement as classical form is impossible. Therefore, over the years of developments of quantum mechanics, always challenging part of quantum mechanics lies in measurements. This book has been written by an international invited group of authors and it is created to clarify different interpretation about measurement in quantum mechanics.

\title{
How to reference
}

In order to correctly reference this scholarly work, feel free to copy and paste the following:

Carsten Held (2012). The Quantum Completeness Problem, Measurements in Quantum Mechanics, Prof. Mohammad Reza Pahlavani (Ed.), ISBN: 978-953-51-0058-4, InTech, Available from:

http://www.intechopen.com/books/measurements-in-quantum-mechanics/the-quantum-completeness-problem

\section{INTECH}

open science | open minds

\section{InTech Europe}

University Campus STeP Ri

Slavka Krautzeka 83/A

51000 Rijeka, Croatia

Phone: +385 (51) 770447

Fax: +385 (51) 686166

www.intechopen.com

\section{InTech China}

Unit 405, Office Block, Hotel Equatorial Shanghai

No.65, Yan An Road (West), Shanghai, 200040, China

中国上海市延安西路65号上海国际贵都大饭店办公楼 405 单元

Phone: +86-21-62489820

Fax: +86-21-62489821 
(C) 2012 The Author(s). Licensee IntechOpen. This is an open access article distributed under the terms of the Creative Commons Attribution 3.0 License, which permits unrestricted use, distribution, and reproduction in any medium, provided the original work is properly cited. 